Internat. J. Math. \& Math. Sci.

Vol. 23, No. 4 (2000) 289-295

S0161171200000466

(C) Hindawi Publishing Corp.

\title{
DOUBLE AND TRIPLE SUMMATION EXPRESSIONS OBTAINED USING PERTURBATION THEORY
}

\author{
HARRY A. MAVROMATIS
}

(Received 4 May 1998)

\begin{abstract}
Using standard perturbation theory, simple new double and triple summation expressions are obtained.
\end{abstract}

Keywords and phrases. Perturbation theory, hypergeometric functions, multiple summation expressions.

2000 Mathematics Subject Classification. Primary 40B05.

1. Introduction. Using standard nondegenerate perturbation theory, if the exact energy of a system is known, we can compare this exact expression to the perturbation expansion of the energy in powers of some small parameter. Equating equal powers of this small parameter is shown to lead, in a conceptually direct way, to new summation expressions with unexpectedly simple values.

2. Theory. In standard perturbation theory [7], it is shown that, given the 3-dimensional system

$$
\begin{gathered}
H=H_{0}+h(r), \\
H_{0}=-\frac{1}{2} \frac{d^{2}}{d r^{2}}+\frac{l(l+1)}{2 r^{2}}+V(r),
\end{gathered}
$$

where $V(r)$ is a central potential and $h(r)$ is a small central perturbation, then

$$
\begin{gathered}
H \Psi_{n l}(r)=E_{n l} \Psi_{n l}(r), \\
H_{0} R_{n l}(r)=E_{n l}^{(0)} R_{n l}(r)=E_{n l}^{(0)}|n l\rangle,
\end{gathered}
$$

and, if $\langle n l|h(r)| n l\rangle=0$, we can write

$$
\begin{aligned}
E_{n l}= & E_{n l}^{(0)}+\sum_{n^{\prime} \neq n}^{\infty} \frac{\left\langle n l|h(r)| n^{\prime} l\right\rangle\left\langle n^{\prime} l|h(r)| n l\right\rangle}{E_{n l}^{(0)}-E_{n^{\prime} l}^{(0)}} \\
& +\sum_{n^{\prime}, n^{\prime \prime} \neq n}^{\infty} \frac{\left\langle n l|h(r)| n^{\prime} l\right\rangle\left\langle n^{\prime} l|h(r)| n^{\prime \prime} l\right\rangle\left\langle n^{\prime \prime} l|h(r)| n l\right\rangle}{\left(E_{n l}^{(0)}-E_{n^{\prime} l}^{(0)}\right)\left(E_{n l}^{(0)}-E_{n^{\prime \prime} l}^{(0)}\right)}
\end{aligned}
$$




$$
\begin{aligned}
& +\sum_{n^{\prime}, n^{\prime \prime}, n^{\prime \prime \prime} \neq n}^{\infty} \frac{\left\langle n l|h(r)| n^{\prime} l\right\rangle\left\langle n^{\prime} l|h(r)| n^{\prime \prime} l\right\rangle\left\langle n^{\prime \prime} l|h(r)| n^{\prime \prime \prime} l\right\rangle\left\langle n^{\prime \prime \prime} l|h(r)| n l\right\rangle}{\left(E_{n l}^{(0)}-E_{n^{\prime} l}^{(0)}\right)\left(E_{n l}^{(0)}-E_{n^{\prime \prime} l}^{(0)}\right)\left(E_{n l}^{(0)}-E_{n^{\prime \prime \prime} l}^{(0)}\right)} \\
& -\sum_{n^{\prime} \neq n}^{\infty} \frac{\left\langle n l|h(r)| n^{\prime} l\right\rangle\left\langle n^{\prime} l|h(r)| n l\right\rangle}{E_{n l}^{(0)}-E_{n^{\prime} l}^{(0)}} \sum_{n^{\prime} \neq n}^{\infty} \frac{\left\langle n l|h(r)| n^{\prime} l\right\rangle\left\langle n^{\prime} l|h(r)| n l\right\rangle}{\left(E_{n l}^{(0)}-E_{n^{\prime} l}^{(0)}\right)^{2}}+\cdots
\end{aligned}
$$

which is a converging series for appropriate $h(r)$ 's. Here, $\left\langle n l|h(r)| n^{\prime} l\right\rangle=\int_{0}^{\infty} R_{n l}(r) \times$ $h(r) R_{n^{\prime} l}(r) r^{2} d r$, etc.

The new summation results obtained are (2.6), (2.9), (2.10), (2.11), and (2.12). Of these, the simplest is

$$
\frac{1}{\beta}=\sum_{n^{\prime} \neq n}^{\infty} \frac{n_{>} ! \Gamma\left(n_{<}+\beta+1\right)}{n_{<} ! \Gamma\left(n_{>}+\beta+1\right)} \frac{1}{\left(n^{\prime}-n\right)}, \quad(\beta \neq 0),
$$

where $n_{>}\left(n_{<}\right)$is the biggest (smallest) of $n, n^{\prime}$. If $\beta$ is an integer, equation (2.6) reduces to

$$
\frac{1}{\beta}=\sum_{n^{\prime} \neq n}^{\infty} \frac{n_{>} !\left(n_{<}+\beta\right) !}{n_{<} !\left(n_{>}+\beta\right) !} \frac{1}{\left(n^{\prime}-n\right)} .
$$

If $n=0$, this reduces to the standard 1-parameter expression [2, p. 11]

$$
\frac{1}{\beta \beta !}=\sum_{k=1}^{\infty} \frac{(k-1) !}{(k+\beta) !} .
$$

The other four expressions are:

$$
\begin{aligned}
& \frac{1}{\beta^{2} \beta !}=\sum_{k=1}^{\infty} \frac{(k-1) !}{(k+\beta) !}\left(\sum_{m=1}^{k-1} \frac{1}{m}\right), \\
& \frac{1}{\beta^{3}} \frac{1}{\beta !}=\sum_{i=1}^{\infty} \frac{(i+\beta) !}{i i !}\left\{\sum_{j>i}^{\infty} \frac{(j-1) !}{(j+\beta) !}\right\}^{2}, \\
& \frac{1}{\beta^{3}} \frac{1}{\beta !}=\sum_{i=1}^{\infty} \frac{1}{i} \sum_{j<i}^{\infty} \frac{1}{j} \sum_{k>i}^{\infty} \frac{(k-1) !}{(k+\beta) !} \\
& \frac{1}{\beta^{3}} \frac{1}{\beta !}=\frac{1}{2} \sum_{i=1}^{\infty} \frac{(i-1) !}{(i+\beta) !}\left\{\left(\sum_{j<i}^{\infty} \frac{1}{j}\right)^{2}-\frac{1}{\beta i}\right\} .
\end{aligned}
$$

If $\beta$ is not an integer, all factorials involving $\beta$ must be replaced by $\gamma$ functions. Thus, (2.9) becomes

$$
\frac{1}{\beta^{2}} \frac{1}{\Gamma(\beta+1)}=\sum_{k=0}^{\infty} \frac{(k-1) !}{\Gamma(k+\beta+1)}\left(\sum_{m=1}^{k-1} \frac{1}{m}\right), \quad(\beta \neq 0),
$$

and so on. 
3. Derivation. Consider the case when in (2.2) $V(r)=(1 / 2) r^{2}$, i.e., we have a 3dimensional harmonic oscillator system. Then [4],

$$
R_{n l}(r)=\left\{\frac{2 \Gamma(n+l+3 / 2)}{n !}\right\}^{1 / 2} \frac{r^{l} e^{-r^{2} / 2}}{\Gamma(l+3 / 2)}{ }_{1} F_{1}\left(-n ; l+\frac{3}{2} ; r^{2}\right)
$$

Here, $n=0,1,2, \ldots, l=0,1,2, \ldots$, and the confluent hypergeometric function [3, p. 1045]

$$
{ }_{1} F_{1}\left(-n ; l+\frac{3}{2} ; r^{2}\right)=\sum_{m=0}^{n} \frac{\Gamma(-n+m) \Gamma(l+3 / 2) r^{2 m}}{\Gamma(-n) \Gamma(l+3 / 2+m) m !}
$$

is an $n+1$-term polynomial in $r^{2}$.

For this $V(r)$, the unperturbed energy in (2.4) is

$$
E_{n l}^{(0)}=2 n+l+\frac{3}{2}
$$

The bit of ingenuity required here is to use, for the central perturbation in (2.1),

$$
h(r)=\alpha\left\{\frac{1}{2 r^{2}}-\frac{1}{2 l+1}\right\}
$$

Then,

$$
H=-\frac{1}{2} \frac{d^{2}}{d r^{2}}+\frac{l(l+1)+\alpha}{2 r^{2}}+\frac{1}{2} r^{2}-\frac{\alpha}{2 l+1}
$$

can be written as

$$
H=-\frac{1}{2} \frac{d^{2}}{d r^{2}}+\frac{l^{\prime}\left(l^{\prime}+1\right)}{2 r^{2}}+\frac{1}{2} r^{2}-\frac{\alpha}{2 l+1},
$$

where

$$
l^{\prime 2}+l^{\prime}-l^{2}-l-\alpha=0
$$

i.e.,

$$
\begin{aligned}
l^{\prime} & =\frac{-1+(2 l+1) \sqrt{1+(4 \alpha) /(2 l+1)^{2}}}{2} \\
& =l+\frac{\alpha}{2 l+1}-\frac{\alpha^{2}}{(2 l+1)^{3}}+\frac{2 \alpha^{3}}{(2 l+1)^{5}}-\frac{5 \alpha^{4}}{(2 l+1)^{7}}+\cdots
\end{aligned}
$$

Thus, for this $h(r)$, the exact energy in (2.3) and (2.5) is known, namely,

$$
E_{n l}=2 n+l^{\prime}+\frac{3}{2}-\frac{\alpha}{2 l+1} .
$$


As is shown below, for this $h(r),\langle n l|h(r)| n l\rangle=0$, for all values of $n$, and we have

$$
\begin{aligned}
& E_{n l}-E_{n l}^{(0)} \\
& =2 n+l^{\prime}+\frac{3}{2}-\frac{\alpha}{2 l+1}-\left(2 n+l+\frac{3}{2}\right)=l^{\prime}-l-\frac{\alpha}{2 l+1} \\
& =-\frac{\alpha^{2}}{(2 l+1)^{3}}+\frac{2 \alpha^{3}}{(2 l+1)^{5}}-\frac{5 \alpha^{4}}{(2 l+1)^{7}}+\cdots \\
& =\alpha^{2} \sum_{n^{\prime} \neq n}^{\infty} \frac{\left\langle n l\left|1 / 2 r^{2}\right| n^{\prime} l\right\rangle\left\langle n^{\prime} l\left|1 / 2 r^{2}\right| n l\right\rangle}{2\left(n-n^{\prime}\right)} \\
& +\alpha^{3} \sum_{\substack{n^{\prime}, n^{\prime \prime} \neq n, n^{\prime} \neq n^{\prime \prime}}}^{\infty} \frac{\left\langle n l\left|1 / 2 r^{2}\right| n^{\prime} l\right\rangle\left\langle n^{\prime} l\left|1 / 2 r^{2}\right| n^{\prime \prime} l\right\rangle\left\langle n^{\prime \prime} l\left|1 / 2 r^{2}\right| n l\right\rangle}{4\left(n-n^{\prime}\right)\left(n-n^{\prime \prime}\right)} \\
& +\alpha^{4}\left\{\sum_{\substack{n^{\prime}, n^{\prime \prime}, n^{\prime \prime \prime} \neq n, n^{\prime} \neq n^{\prime \prime}, n^{\prime \prime} \neq n^{\prime \prime \prime}}}^{\infty} \frac{\left\langle n l\left|1 / 2 r^{2}\right| n^{\prime} l\right\rangle\left\langle n^{\prime} l\left|1 / 2 r^{2}\right| n^{\prime \prime} l\right\rangle\left\langle n^{\prime \prime} l\left|1 / 2 r^{2}\right| n^{\prime \prime \prime} l\right\rangle\left\langle n^{\prime \prime \prime} l\left|1 / 2 r^{2}\right| n l\right\rangle}{2\left(n-n^{\prime}\right) 2\left(n-n^{\prime \prime}\right) 2\left(n-n^{\prime \prime \prime}\right)}\right. \\
& \left.-\sum_{n^{\prime} \neq n}^{\infty} \frac{\left\langle n l\left|1 / 2 r^{2}\right| n^{\prime} l\right\rangle\left\langle n^{\prime} l\left|1 / 2 r^{2}\right| n l\right\rangle}{2\left(n-n^{\prime}\right)} \sum_{n^{\prime} \neq n}^{\infty} \frac{\left\langle n l\left|1 / 2 r^{2}\right| n^{\prime} l\right\rangle\left\langle n^{\prime} l\left|1 / 2 r^{2}\right| n l\right\rangle}{4\left(n-n^{\prime}\right)^{2}}\right\}+\cdots
\end{aligned}
$$

Then we can equate equal powers of $\alpha$ to obtain

$$
\begin{aligned}
& \frac{1}{(2 l+1)^{3}}=\sum_{n^{\prime} \neq n}^{\infty} \frac{\left\langle n l\left|1 / 2 r^{2}\right| n^{\prime} l\right\rangle\left\langle n^{\prime} l\left|1 / 2 r^{2}\right| n l\right\rangle}{2\left(n^{\prime}-n\right)} \\
& \frac{1}{(2 l+1)^{5}}=\sum_{\substack{n^{\prime}, n^{\prime \prime} \neq n, n^{\prime} \neq n^{\prime \prime}}}^{\infty} \frac{\left\langle n l\left|1 / 2 r^{2}\right| n^{\prime} l\right\rangle\left\langle n^{\prime} l\left|1 / 2 r^{2}\right| n^{\prime \prime} l\right\rangle\left\langle n^{\prime \prime} l\left|1 / 2 r^{2}\right| n l\right\rangle}{8\left(n-n^{\prime}\right)\left(n-n^{\prime \prime}\right)} \\
& \frac{1}{(2 l+1)^{7}} \\
& =\frac{1}{5}\left\{\begin{array}{l}
\sum_{\substack{n^{\prime}, n^{\prime \prime}, n^{\prime \prime \prime} \neq n, n^{\prime} \neq n^{\prime \prime}, n^{\prime \prime} \neq n^{\prime \prime \prime}}} \frac{\left\langle n l\left|1 / 2 r^{2}\right| n^{\prime} l\right\rangle\left\langle n^{\prime} l\left|1 / 2 r^{2}\right| n^{\prime \prime} l\right\rangle\left\langle n^{\prime \prime} l\left|1 / 2 r^{2}\right| n^{\prime \prime \prime} l\right\rangle\left\langle n^{\prime \prime \prime} l\left|1 / 2 r^{2}\right| n l\right\rangle}{2\left(n-n^{\prime}\right) 2\left(n-n^{\prime \prime}\right) 2\left(n-n^{\prime \prime \prime}\right)} \\
\left.-\sum_{n^{\prime} \neq n}^{\infty} \frac{\left\langle n l\left|1 / 2 r^{2}\right| n^{\prime} l\right\rangle\left\langle n^{\prime} l\left|1 / 2 r^{2}\right| n l\right\rangle}{2\left(n-n^{\prime}\right)} \sum_{n^{\prime} \neq n}^{\infty} \frac{\left\langle n l\left|1 / 2 r^{2}\right| n^{\prime} l\right\rangle\left\langle n^{\prime} l\left|1 / 2 r^{2}\right| n l\right\rangle}{4\left(n-n^{\prime}\right)^{2}}\right\}
\end{array}\right.
\end{aligned}
$$

To evaluate (3.11), (3.12), and (3.13) we can use the general expression [5] 


$$
\begin{aligned}
I_{n n^{\prime} l}(\lambda)= & \left\langle n l\left|r^{\lambda}\right| n^{\prime} l\right\rangle \\
= & {\left[\frac{\Gamma(n+l+3 / 2)}{n ! n^{\prime} ! \Gamma\left(n^{\prime}+l+3 / 2\right)}\right]^{1 / 2} \frac{\Gamma(\lambda / 2+l+3 / 2) \Gamma\left(n^{\prime}-\lambda / 2\right)}{\Gamma(l+3 / 2) \Gamma(-\lambda / 2)} } \\
& \times{ }_{3} F_{2}\left(-n, \frac{\lambda}{2}+l+\frac{3}{2}, \frac{\lambda}{2}+1 ; l+\frac{3}{2},-n^{\prime}+\frac{\lambda}{2}+1 ; 1\right), \quad\left(n^{\prime} \geq n\right),
\end{aligned}
$$

where the generalized hypergeometric function ${ }_{3} F_{2}$ is an $n+1$-term polynomial [3, p. 1045], namely,

$$
\begin{gathered}
=\sum_{p=0}^{n} \frac{\Gamma(-n+p) \Gamma(\lambda / 2+l+3 / 2+p) \Gamma(\lambda / 2+l+p)}{\Gamma(-n) \Gamma(\lambda / 2+l+3 / 2) \Gamma(\lambda / 2+1)} \\
\quad \times \frac{\Gamma(l / 2+3 / 2) \Gamma\left(-n^{\prime}+\lambda / 2+1\right)}{\Gamma(l / 2+3 / 2+p) \Gamma\left(-n^{\prime}+\lambda / 2+1+p\right) p !} .
\end{gathered}
$$

We note that if any of the terms $-n, \lambda / 2+l+3 / 2, \lambda / 2+1$ is zero, the ${ }_{3} F_{2}$ in (3.14) is equal to 1 .

The integral expression equation (3.14) (with $n=n^{\prime}, \lambda=-2$ ) confirms that $\langle n l|h(r)|$ $n l\rangle=0$ since

$$
\begin{aligned}
\left\langle n l\left|1 / 2 r^{2}\right| n l\right\rangle & =\frac{1}{2} \frac{1}{n !} \frac{\Gamma(l+1 / 2) \Gamma(n+1)}{\Gamma(l+3 / 2) \Gamma(1)}{ }_{3} F_{2}\left(-n, l+\frac{1}{2}, 0 ; l+\frac{3}{2},-n ; 1\right) \\
& =\frac{1}{2} \frac{1}{(l+1 / 2)},
\end{aligned}
$$

where we have used $\Gamma(\beta+1)=\beta \Gamma(\beta)$, and $\Gamma(m+1)=m$ ! if $m$ is an integer. Hence,

$$
\left\langle n l\left|\left\{\frac{1}{2 r^{2}}-\frac{1}{2 l+1}\right\}\right| n l\right\rangle=0 .
$$

This result was recently discussed in an interesting paper [1], where it is obtained using the Hellman-Feynman theorem. Here, this result is shown to also follow from (3.14) (with $n=n^{\prime}$, and $\lambda=-2$ ). We note a very interesting property of this result, namely, that $\left\langle n l\left|1 / 2 r^{2}\right| n l\right\rangle$ is independent of the variable $n$.

Equation (3.11) gives the simplest new summation expression. Using (3.14) again (this time for $n \neq n^{\prime}$ ), we obtain

$$
\begin{aligned}
\left\langle n l\left|1 / 2 r^{2}\right| n^{\prime} l\right\rangle= & \frac{1}{2}\left[\frac{\Gamma(n+l+3 / 2)}{n ! n^{\prime} ! \Gamma\left(n^{\prime}+l+3 / 2\right)}\right]^{1 / 2} \frac{\Gamma(l+1 / 2) \Gamma\left(n^{\prime}+1\right)}{\Gamma(l+3 / 2) \Gamma(1)} \\
& \times{ }_{3} F_{2}\left(-n, l+\frac{1}{2}, 0 ; l+\frac{3}{2},-n^{\prime} ; 1\right) \\
= & \frac{1}{2}\left(\frac{n^{\prime} ! \Gamma(n+l+3 / 2)}{n ! \Gamma\left(n^{\prime}+l+3 / 2\right)}\right)^{1 / 2} \frac{1}{(l+1 / 2)}, \quad\left(n^{\prime}>n\right) .
\end{aligned}
$$

Hence,

$$
\frac{1}{(2 l+1)^{3}}=\frac{1}{8} \sum_{n^{\prime} \neq n} \frac{n_{>} ! \Gamma\left(n_{<}+l+3 / 2\right)}{n_{<} ! \Gamma\left(n_{>}+l+3 / 2\right)} \frac{1}{(l+1 / 2)^{2}\left(n^{\prime}-n\right)},
$$

where $n_{>},\left(n_{<}\right)$is the biggest (smallest) of $n, n^{\prime}$. This is just a result of (2.6) if we substitute $\beta=l+1 / 2$. 
By comparing the next power of alpha $\left(\alpha^{3}\right)$ in (3.12), we obtain a more complicated new double sum expression. For the special case $n=0$, this reduces to

$$
\frac{1}{\beta^{2}} \frac{1}{\Gamma(\beta+1)}=\sum_{k=1}^{\infty} \frac{(k-1) !}{\Gamma(k+\beta+1)}\left(\sum_{m=1}^{k-1} \frac{1}{m}\right), \quad(\beta \neq 0) .
$$

This new result can be added to the known series of the form [6, p. 695]

$$
\sum a_{k}\left(\sum^{N(k)} b_{m}\right)
$$

If $\beta$ is an integer, equation (3.20) reduces to

$$
\frac{1}{\beta^{2}} \frac{1}{\beta !}=\sum_{k=1}^{\infty} \frac{(k-1) !}{(k+\beta) !}\left(\sum_{m=1}^{k-1} \frac{1}{m}\right) .
$$

Finally, comparing the fourth power of $\alpha$, we obtain, for the simple case $n=0, \beta=$ integer, 3 triple sum results, namely,

$$
\begin{aligned}
& \frac{1}{\beta^{3}} \frac{1}{\beta !}=\sum_{i=1}^{\infty} \frac{(i+\beta) !}{i i !}\left\{\sum_{j>i}^{\infty} \frac{(j-1) !}{(j+\beta) !}\right\}^{2}, \\
& \frac{1}{\beta^{3}} \frac{1}{\beta !}=\sum_{i=1}^{\infty} \frac{1}{i} \sum_{j<i}^{\infty} \frac{1}{j} \sum_{k>i}^{\infty} \frac{(k-1) !}{(k+\beta) !} \\
& \frac{1}{\beta^{3}} \frac{1}{\beta !}=\frac{1}{2} \sum_{i=1}^{\infty} \frac{(i-1) !}{(i+\beta) !}\left\{\left(\sum_{j<i}^{\infty} \frac{1}{j}\right)^{2}-\frac{1}{\beta i}\right\} .
\end{aligned}
$$

4. Conclusions. We have used a term-by-term comparison between the exact expression for the energy of a particular system and the perturbation expansion of this energy in powers of a parameter to obtain new, multiple summation expressions. The results of the summations are remarkably simple given the multiple summations involved. All these expressions were verified for a few specific values of $\beta$ using the Mathematica program [8, p. 69]. One expects that continuing, as above, to higher orders, one can obtain quadruple sums related to $1 /\left(\beta^{4} \beta\right.$ !), etc.

ACKNOWLEDGEMENT. The author would like to acknowledge KFUPM support.

\section{REFERENCES}

[1] H. Beker, A simple calculation of $1 / r^{2}$ for the Hydrogen atom and the 3-dimensional harmonic oscillator, Amer. J. Phys. 65 (1997), 1118-1119.

[2] I. S. Gradshteyn and I. M. Ryzhik, Table of integrals, series, and products, 4th ed., Academic Press, New York, London, Toronto, 1980, prepared by A. Jeffrey. MR 81g:33001. Zbl 521.33001 .

[3] _ Table of integrals, series, and products, 4th ed., Academic Press, New York, London, Toronto, 1980, prepared by A. Jeffrey. MR 81g:33001. Zbl 521.33001.

[4] H. Mavromatis, Exercises in quantum mechanics, 2d ed., Kluwer Texts in the Mathematical Sciences, vol. 6, Kluwer Academic Publishers Group, Dordrecht, 1992, A collection of illustrative problems and their solutions. MR 92k:81003. Zbl 821.00006. 
[5] H. A. Mavromatis, Oscillator-Basis Electric Multiple Selection Rules, Proceedings International Nucler Physics Conference (Greece), thessaloniki, 1997, to be published.

[6] A. P. Prudnikov, Yu. A. Brychkov, and O. I. Marichev, Integrals and series. Vol. 1, Gordon \& Breach Science Publishers, New York, 1986, Elementary functions. MR 88f:00014. Zbl 733.00004 .

[7] J. S. Townsend, A Modern Aapproach to Quantum Mechanics, McGraw-Hill, 1992, p. 309310.

[8] S. Wolfram, Mathematica: A system for doing mathematics by computer, Addison-Wesley Publishing Company, Inc., Reading, M.A., 1988. Zbl 671.65002.

Mavromatis: Physics DePartment, King Fahd University of Petroleum AND Minerals, DHAHRAN 31261, SAUDI ARABIA 


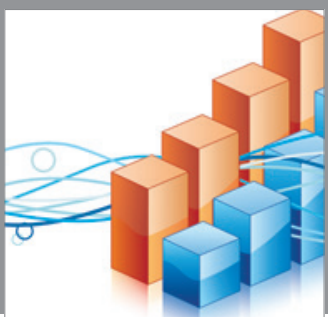

Advances in

Operations Research

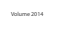

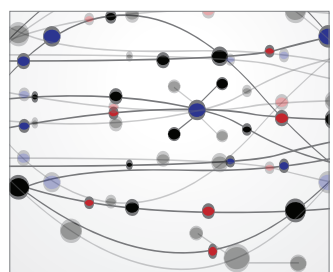

\section{The Scientific} World Journal
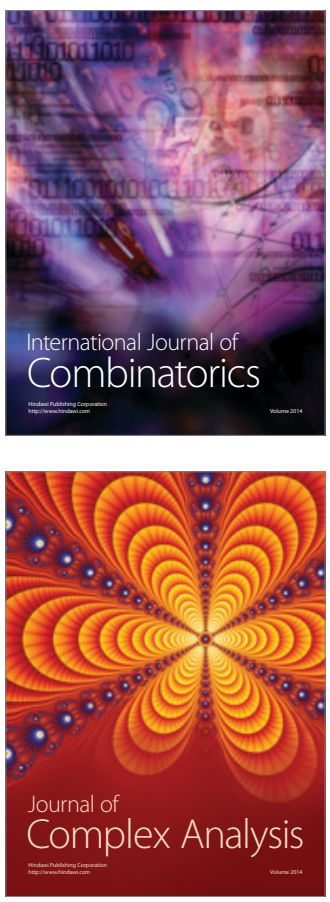

International Journal of

Mathematics and

Mathematical

Sciences
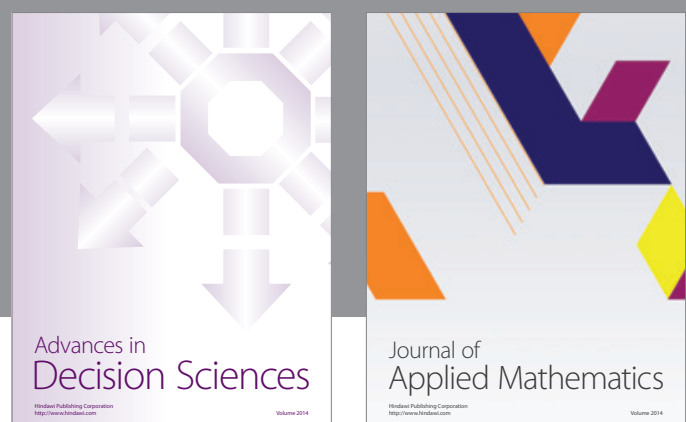

Journal of

Applied Mathematics
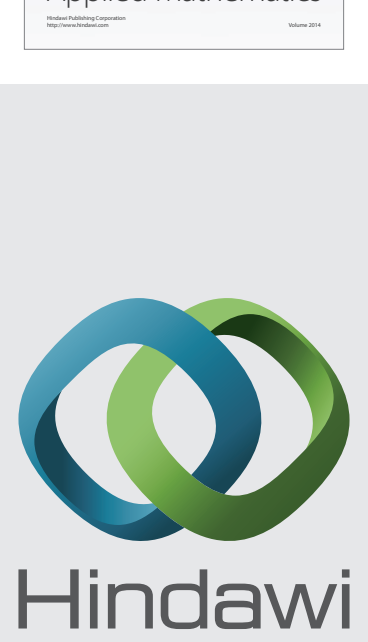

Submit your manuscripts at http://www.hindawi.com
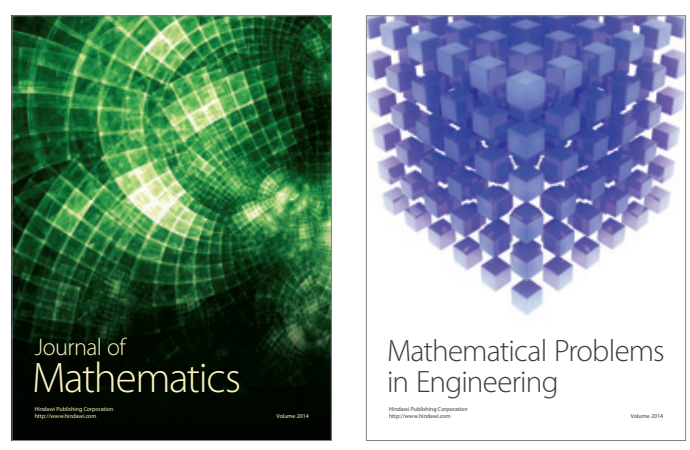

Mathematical Problems in Engineering
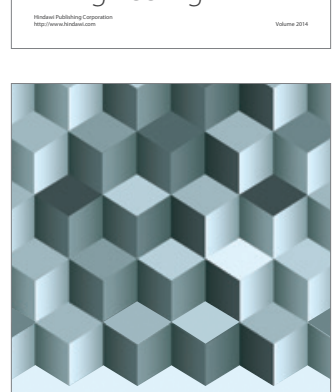

Journal of

Function Spaces
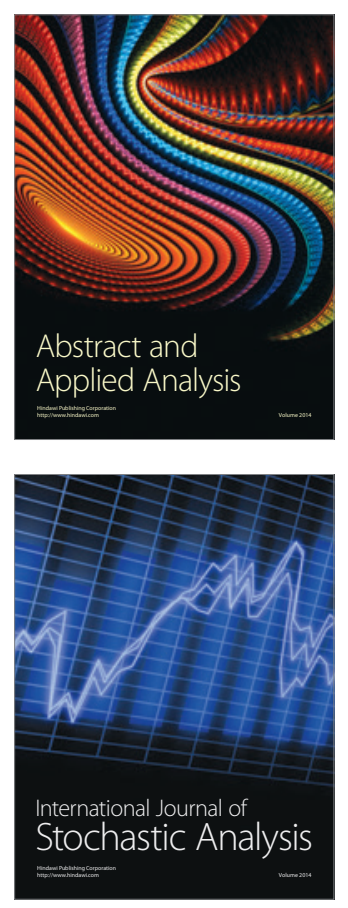

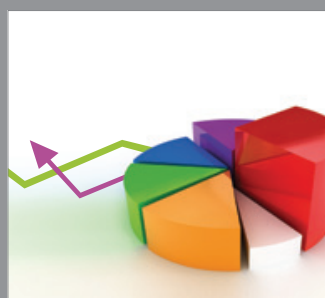

ournal of

Probability and Statistics

Promensencen
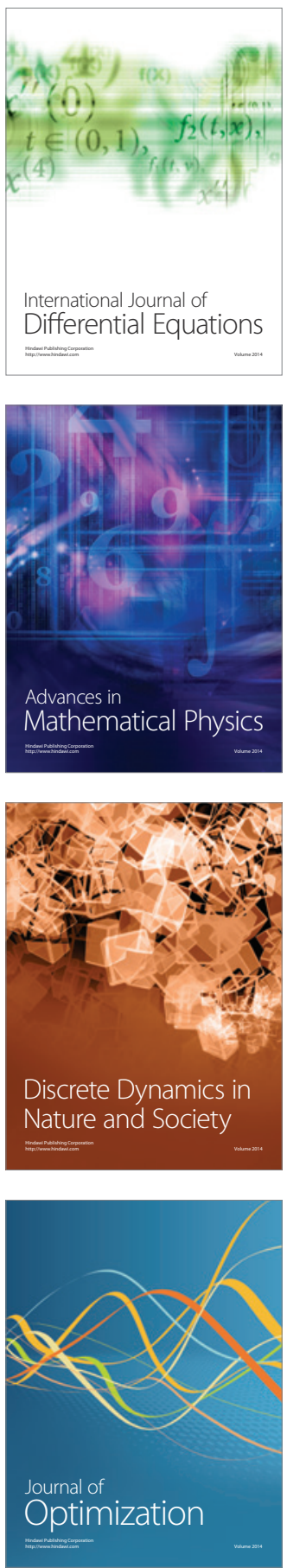